\title{
ÉTUDE THÉORIQUE DE LA RELAXATION D'ATOMES ALGALINS PAR GOLLISIONS SUR UNE PAROI ET SUR UN GAZ
}

\author{
Par Françoise MASNOU-SEEUWS, Marie-Anne BOUGHIAT.
}

\begin{abstract}
Résumé. - L'étude théorique présentée a pour but de calculer l'évolution dans le temps de la valeur moyenne d'une observable $Q$ relative à un ensemble d'atomes alcalins dans l'état fondamental, lorsque la relaxation a pour cause les deux processus suivants : d'une part les collisions contre la paroi après diffusion dans le gaz, d'autre part les collisions des atomes alcalins contre les atomes du gaz. Le calcul utilise le formalisme de la matrice densité, ce qui permet de calculer l'évolution de différentes observables et de tenir compte de ce que des observables différentes peuvent relaxer sur la paroi ou sur le gaz avec des constantes de temps différentes. La mise en équation est valable pour une paroi recouverte d'un enduit (collisions faibles de type magnétique) et pour une paroi très désorientante (verre nu ou recouvert d'un miroir alcalin).

Cette étude doit permettre d'interpréter les résultats expérimentaux et d'en déduire le coefficient de diffusion de l'alcalin dans le gaz étranger et les sections efficaces de désorientation (alcalin-gaz étranger) relatives à diverses observables. Les rapports entre ces derniers paramètres apportent des renseignements quant à la nature de l'interaction désorientatrice en phase gazeuse et au caractère du spectre de ses fluctuations.
\end{abstract}

Abstract. - The time dependence of the mean value of an observable $Q$ (measured over an ensemble of alkali atoms in the ground state) is theoretically studied when two relaxation processes are simultaneously present : collisions against the walls of the cell, after diffusion through a foreign gas and collisions with the molecules of the gas. The use of the density matrix formalism makes it possible to calculate the time evolution of different observables taking into account the fact that their relaxation in the gas phase and on the walls can exhibit different time constants. The present theory is valid for a coated wall (weak collisions of the magnetic type) or for an uncoated wall (strong collisions). It should lead to an interpretation of experimental results and allow a determination of the diffusion constant of alkali atoms in a buffer gas and of the deorientation cross sections for different observables. The ratios between these cross sections give information on the physical nature of the disorientating interaction in the gas phase and on its fluctuation spectrum.

I. Introduction. - L'expérience a montré [1] que toute collision d'un atome alcalin dans l'état fondamental sur une paroi de verre nue produit la désorientation de son spin $\mathbf{S}$. Aux pressions de vapeur auxquelles on produit l'orientation par pompage optique, le libre parcours moyen est grand devant les dimensions de la cellule, par suite le temps de relaxation est le temps qui sépare deux collisions successives sur la paroi, soit de l'ordre de $10^{-4} \mathrm{~s}$. Il existe deux méthodes permettant d'allonger les temps de relaxation. La première consiste à recouvrir la paroi de la cellule d'un enduit de paraffine saturée. On trouve que 1000 à 10000 collisions sur un enduit de ce type sont nécessaires pour produire la désorientation du spin $\mathbf{S}$ et du spin nucléaire I d'un atome alcalin couplés statiquement par interaction hyperfine. L'étude théorique et expérimentale de la relaxation $d u R b$ sur un tel enduit [2], [3] a montré que la désorientation est due à une interaction aléatoire faible et de type magnétique $\gamma_{\mathrm{S}} \mathbf{S} . \mathbf{H}(t) \quad(\mathbf{H}(t)$ étant le champ magnétique aléatoire vu par l'atome au cours des collisions sur la paroi et satisfaisant à la condition de moyennage par le mouvement [4]). L'origine de l'interaction est en partie la suivante. Les spins nucléaires $\mathbf{K}$ des protons de la paraffine créent au voisinage de la paroi un champ magnétique. Du fait de son mouvement, un atome de spin $\mathbf{S}$ est soumis à une perturbation $\gamma_{\mathrm{S}} \mathbf{S} . \mathbf{H}(t)$ fonction aléatoire du temps. Si dans le spectre de Fourier de cette perturbation se trouve l'une de ses fréquences propres, une transition peut être induite, entraînant la désorientation de $\mathbf{S}$ et par suite de I. Le deuton ayant un moment magnétique nucléaire plus faible que celui du proton, la valeur quadratique moyenne de $\mathbf{H}(t)$ est considérablement réduite si l'on substitue à une paraffine usuelle une paraffine deutérée ; on observe bien dans ce cas une relaxation plus lente [3].

La seconde méthode utilisée pour protéger $\mathbf{S}$ de l'action désorientatrice des parois consiste à opérer en présence d'un gaz étranger ou " gaz tampon ». Si le 
libre parcours moyen de l'atome alcalin est petit devant les dimensions de la cellule, l'influence désorientatrice de la paroi est nettement réduite pour les atomes de $\mathrm{Rb}$ situés en son centre et diffusant lentement vers les bords. Cependant, les collisions de l'alcalin sur les atomes de gaz tampon sont elles-mêmes une cause de relaxation. Pour les divers gaz rares, la section efficace de désorientation est très faible ; il a été montré [5] qu'il faut $10^{4}$ à $10^{8}$ collisions pour désorienter un atome de $\mathrm{Rb}$. Une étude théorique [6], [7] aboutit à la conclusion qu'il existe des perturbations aléatoires faibles de type magnétique dont l'ordre de grandeur permettrait d'expliquer les temps de relaxation observés en phase gazeuse. Pour les gaz rares qui n'ont pas de spin nucléaire, il s'agit de l'interaction « spinorbite " $\mathbf{S} . \mathbf{N}$ où $\mathbf{N}$ représente le moment cinétique orbital relatif des atomes alcalin-gaz rare entrant en collision [6]. Dans le cas où le gaz rare possède un spin nucléaire K, la théorie d'Herman [7] prévoit que l'interaction magnétique entre $\mathbf{S}$ et $\mathbf{K}$ est négligeable devant l'interaction $\mathbf{S}$. $\mathbf{N}$, sauf dans le cas de ${ }^{3} \mathrm{He}$ et ${ }^{21} \mathrm{Ne}$.

Il nous a paru intéressant de reprendre l'étude théorique de la relaxation sous l'effet simultané des collisions sur la paroi et sur le gaz malgré le nombre de travaux antérieurs déjà relatifs à ce sujet. Les raisons en sont les suivantes :

- Jusqu'ici les calculs théoriques publiés [8] ont seulement envisagé le cas d'une paroi totalement désorientante. Celui que nous exposons ici est aussi valable si les parois de la cellule sont recouvertes d'un enduit de bonne qualité.

- Les calculs antérieurs supposent également que la relaxation sur le gaz peut être caractérisée par une seule constante de temps. Or il a été montré [2] que, sous l'effet d'une interaction faible aléatoire de type magnétique, la relaxation des diverses observables d'un alcalin de spin nucléaire I fait intervenir un grand nombre de constantes de temps : il existe par exemple $(4 I+1)$ constantes de temps longitudinales dont les rapports mutuels sont compris entre 1 et $(2 I+1)^{2}$. Le présent calcul est fait dans le formalisme de la matrice densité et tient compte de cet effet à la fois pour les collisions sur le gaz et sur la paroi.

L'interprétation des résultats expérimentaux dans le cadre de la présente théorie devrait permettre d'éviter l'énorme dispersion que l'on note actuellement dans la littérature entre les valeurs des sections efficaces de désorientation alcalin-gaz rare déterminées par des expérimentateurs différents ; en effet, cette dispersion tient très probablement en partie à l'ambiguïté qui règne quant à l'observable étudiée par chacun d'entre eux.

II. Mise en équation du problème. - Étant donnée une grandeur physique à laquelle est associé un opérateur $Q$, nous nous proposons d'étudier l'évolution dans le temps de $\langle Q\rangle$, valeur moyenne de l'observable $Q$ prise sur l'ensemble des atomes alcalins (dans l'état fondamental) et occupant un certain volume $V_{\mathrm{d}}$ (le volume de vapeur analysé par le procédé de détection). Nous supposons que cette évolution est produite par les quatre causes suivantes :

1) Hamiltonien statique $\mathscr{H}_{0}$ : couplage hyperfin $a \mathbf{S}$. I et interaction Zeeman;

2) Diffusion dans le gaz étranger;

3) Relaxation par collisions sur la paroi;

4) Relaxation par collisions sur le gaz.

Nous ne tenons compte ni de l'effet du faisceau lumineux ni de l'effet des collisions d'échange des atomes alcalins entre eux. (Les valeurs expérimentales des temps de relaxation qui nous intéressent sont déduites de mesures effectuées " dans le noir " après extrapolation à la valeur nulle de la tension de vapeur d'alcalin.)

Nous allons considérer séparément l'effet de chacun de ces processus puis l'effet global.

1. Évolution sous L'EFFET DE $\mathscr{H}_{0}$. - Nous supposons que $\mathscr{H}_{0}$ est le même en tous les points du volume $V$ où sont confinés les atomes : nous négligeons les effets liés à l'inhomogénéité $d u$ champ $H_{0}$. Dans ces conditions, une observable évolue d'une part sous l'effet de $\mathscr{H}_{0}$, et, d'autre part, sous l'effet du mouvement des atomes ; ces deux évolutions sont indépendantes $(\mathbf{1})$.

Nous pouvons passer en représentation d'interaction de manière à éliminer l'évolution rapide (liée à $\mathscr{H}_{0}$ ) des éléments de la matrice densité de l'ensemble de tous les atomes $\sigma(t)$. Le passage de cette représentation, $\sigma^{*}$, à celle du laboratoire, $\sigma$, se fait au moyen des formules :

$$
\begin{aligned}
\sigma(t) & =\mathrm{e}^{-i \mathscr{H}_{0} t} \sigma^{*}(t) \mathrm{e}^{i \mathscr{H}_{0} t} \\
\frac{\mathrm{d} \sigma}{\mathrm{d} t} & =\mathrm{e}^{-i \mathscr{H}_{0} t} \frac{\mathrm{d} \sigma^{*}}{\mathrm{~d} t} \mathrm{e}^{i \mathscr{H}_{0} t}-i\left[\mathscr{H}_{0}, \sigma\right] .
\end{aligned}
$$

On montre que l'évolution de $\sigma^{*}$ se calcule simplement en omettant $\mathscr{H}_{0}$ parmi les quatre causes d'évolution citées ci-dessus.

2. Diffusion dans le gaz Étranger (en l'absence de relaxation sur ce dernier). - La densité d'atomes alcalins en un point est une fonction de $\mathbf{r}$ et de $t$, $n(\mathbf{r}, t)$ dont l'évolution dans le temps obéit à l'équation de diffusion :

$$
\frac{\mathrm{d}}{\mathrm{d} t} n(\mathbf{r}, t)=D \nabla^{2} n(\mathbf{r}, t) .
$$

Nous nous intéresserons plus généralement à l'effet du mouvement sur l'évolution d'une grandeur phy-

(1) Le couplage entre les évolutions dues au hamiltonien statique et au mouvement de diffusion est essentiel au contraire dans le cas d'une assemblée de spins soumis à des pulses de radiofréquence, tandis qu'ils diffusent dans un champ statique inhomogène. La théorie faite en ajoutant dans l'équation de Bloch un terme décrivant la diffusion montre que les échos de spin peuvent permettre de mesurer le coefficient de diffusion [9]. 
sique quelconque. Considérons un petit élément de volume $\mathrm{d} V$ centré au point $\mathbf{r}$ et l'ensemble des $p$ atomes $(p=n \mathrm{~d} V \gg 1)$ de cet élément ayant pour matrices densités individuelles $\sigma_{1}, \sigma_{2}, \ldots, \sigma_{p}\left(\sigma_{1}^{*}\right.$, $\sigma_{2}^{*}, \ldots, \sigma_{p}^{*}$ en représentation d'interaction). Nous pouvons définir une matrice densité relative à cet ensemble d'atomes :

$$
\sigma^{*}(\mathbf{r}, t)=\frac{1}{p}\left(\sigma_{1}^{*}+\sigma_{2}^{*}+\ldots+\sigma_{p}^{*}\right)
$$

et introduire la valeur moyenne locale de l'observable $Q^{*}$ au point $\mathbf{r}$, qui représente la valeur moyenne de l'observable $Q^{*}$ calculée à un instant $t$ sur l'ensemble des $p$ atomes qui occupent l'élément de volume $\mathrm{d} V$ à cet instant :

$$
\left\langle Q^{*}(\mathbf{r}, t)\right\rangle=\operatorname{Tr}\left\{Q \sigma^{*}(\mathbf{r}, t)\right\} .
$$

Supposons l'élément de volume $\mathrm{d} V$ centré au point $\mathbf{r}$ délimité par deux surfaces planes $\mathrm{d} s$ parallèles (d'abscisses $x$ et $x+\mathrm{d} x)$ et considérons l'évolution de $\sigma^{*}(x, t)$ qu'entraîne le seul effet de diffusion des atomes alcalins dans le gaz étranger suivant la direction $O x$. Nous connaissons à l'instant $t$ le flux d'atomes traversant les plans d'abscisses $x$ et $x+\mathrm{d} x$ et entrant dans l'élément de volume considéré :

$$
-D \mathrm{~d} s \frac{\partial}{\partial x} n(x, t) \quad \text { et } \quad+D \mathrm{~d} s \frac{\partial}{\partial x} n(x+\mathrm{d} x) .
$$

La nouvelle valeur prise par la matrice densité à l'instant $t+\mathrm{d} t$ s'évalue en considérant le nouvel ensemble d'atomes occupant l'élément de volume $\mathrm{d} V$ à cet instant. Le calcul se fait simplement, car dans les hypothèses où nous nous plaçons la contribution de chaque atome ne dépend que de sa matrice densité à l'instant $t$, elle est indépendante de l'histoire qui lui est advenue à des instants antérieurs : d'une part, l'hypothèse d'un champ $H_{0}$ homogène nous a permis de nous placer en représentation d'interaction, d'autre part, les collisions sur le gaz sont des collisions faibles. Ceci nous permet d'écrire :

$\frac{\partial}{\partial t} \sigma^{*}(x, t) \mathrm{d} s \mathrm{~d} x=D \mathrm{~d} s \frac{\partial}{\partial x}\left[\sigma^{*}(x+\mathrm{d} x, t)-\sigma^{*}(x, t)\right]$

qui se généralise facilement au cas où l'on s'intéresse à la diffusion dans les 3 directions de l'espace :

$$
\frac{\partial}{\partial t} \sigma^{*}(\mathbf{r}, t)=D \nabla^{2} \sigma^{*}(\mathbf{r}, t) \text {. }
$$

On peut obtenir de manière analogue l'équation d'évolution, sous le seul effet de la diffusion, pour la valeur moyenne locale de l'observable $Q^{*}$ :

$$
\frac{\mathrm{d}}{\mathrm{d} t}\left\langle Q^{*}(\mathbf{r}, t)\right\rangle=D \nabla^{2}\left\langle Q^{*}(\mathbf{r}, t)\right\rangle
$$

$D$ est le coefficient de diffusion de l'alcalin dans le gaz étranger. Si la masse moléculaire du gaz $M_{2}$ est voisine de celle de l'alcalin $M_{1}, D$ est donné par l'expression classique :

$$
D_{\mathrm{cl}}=\lambda \bar{v} / 3
$$

$\lambda$ libre parcours moyen de l'alcalin dans le gaz; $\bar{v}$ vitesse moyenne de l'alcalin.

Dans le cas contraire, la grande différence de masse entre les particules diffusantes et celles du milieu diffuseur rend le processus anisotrope : on peut montrer [10] que la valeur moyenne du cosinus de l'angle $\theta$ entre les directions de la trajectoire de la particule avant et après la collision n'est pas nulle. Si on suppose la diffusion isotrope dans le système du centre de masse, l'anisotropie apparaît lorsqu'on passe de ce système à celui du laboratoire et elle est d'autant plus grande que le rapport $m=M_{2} / M_{1}$ est petit devant 1. Fermi a montré que le mouvement des particules diffusantes peut être dans ce cas encore décrit par une équation de diffusion avec un coefficient de diffusion :

$$
D=D_{\mathrm{cl}} /(1-\overline{\cos \theta})=\lambda_{t} \bar{v} / 3 .
$$

Ceci revient à dire qu'au bout de $1 /(1-\overline{\cos \theta})$ collisions on peut considérer que le processus de diffusion devient isotrope. $1 /(1-\overline{\cos \theta})$ représente le nombre de collisions que doit subir l'atome alcalin pour être dévié dans une direction non corrélée avec sa direction initiale. L'équation (3) définit le libre parcours moyen de transport $\lambda_{t}=\lambda /(1-\overline{\cos \theta})$ qui s'interprète comme la distance moyenne que doit parcourir un atome pour être dévié de sa trajectoire d'un angle arbitraire.

Le calcul de $\overline{\cos \theta}$ a été fait par Cohen-Tannoudji dans le cas où les particules diffusantes et celles du milieu diffuseur sont animées de vitesse obéissant à la distribution maxwellienne de la théorie cinétique des gaz [11]. Nous rappelons ici le résultat :

pour $m \gg 1$

$$
\frac{1}{1-\overline{\cos \theta}} \approx 1+\frac{4}{3 m}
$$

pour $m \ll 1$

$\frac{1}{1-\overline{\cos \theta}}=\left(2 m-\frac{8}{\pi} \frac{16}{15} m \sqrt{m}+\frac{5}{3} m^{2}+\ldots\right)^{-1}$

pour $m=1$

$$
\frac{1}{1-\overline{\cos \theta}}=0,55 \text {. }
$$

On voit en particulier que pour $m \ll 1, \lambda_{t}$ diffère beaucoup de $\lambda$.

Notons que, pour que l'équation de diffusion ait un sens, il faut que $\lambda_{t}$ soit petit devant les dimensions du volume $V$.

3. Relaxation PAR GOllisions SUR LA PAROI. - Il est utile de rappeler tout d'abord quelques résultats antérieurs obtenus au cours de l'étude théorique et 
expérimentale de la relaxation par collisions sur une paroi recouverte d'un enduit paraffiné, en l'absence de gaz.

3.a. Relaxation par collisions sur la paroi en l'absence de gaz. - Pour un enduit paraffiné non contaminé, l'interaction désorientatrice est une interaction aléatoire faible de type magnétique [3]. La théorie [2] fournit l'équation d'évolution de $\sigma^{*}$; elle est complexe quand on tient compte du spin nucléaire $I$ de l'alcalin. Par contre, nous ferons usage du résultat simple suivant : certaines observables $Q_{\mathrm{i}}$ ont leur évolution caractérisée par une constante de temps $T_{i}$ unique, quel que soit l'état initial à partir duquel s'effectue la relaxation :

$$
\frac{\mathrm{d}}{\mathrm{d} t}\left\langle Q_{i}\right\rangle^{*}=-\frac{1}{T_{i}}\left(\left\langle Q_{i}\right\rangle^{*}-\left\langle Q_{i}\right\rangle_{\mathrm{B}}^{*}\right)
$$

$\left(\left\langle Q_{i}\right\rangle_{\mathbf{B}}^{*}\right.$ valeur moyenne de $Q_{i}^{*}$ à l'équilibre de Boltzmann; par la suite, nous supposons les observables $Q_{i}$ définies de façon telle que $\left\langle Q_{i}\right\rangle_{\mathbf{B}}^{*}=0$ ).

C'est par exemple le cas des observables S. I (écart par rapport à l'équilibre de Boltzmann de la différence de populations entre niveaux hyperfins), $I_{z}$ polarisation nucléaire longitudinale et

$$
Q_{\mathrm{e}}=S_{z}-\frac{2}{(2 I+1)^{2}-2} I_{z} .
$$

Notons qu'il y a en tout $(4 I+1)$ observables $Q_{i}$ si on se limite aux grandeurs longitudinales et que n'importe quelle grandeur longitudinale représentée par une observable $Q$ peut se mettre de manière unique sous la forme d'une combinaison linéaire des observables $Q_{i}$

$$
Q=\sum_{i=1}^{4 I+1} a_{i} Q_{i}
$$

Par exemple $S_{z}$ n'est pas une observable $Q_{i}$ : c'est une combinaison linéaire de $Q_{\mathrm{e}}$ et de $I_{z}$.

Pour les observables $Q_{i}$ obéissant à l'équation d'évolution [4], nous pouvons définir une probabilité de «désorientation " par collision sur la paroi :

$$
\alpha_{i}=\tau_{\mathrm{v}} / T_{i}
$$

$\alpha_{i}$ représente l'inverse du nombre de collisions nécessaires pour que l'écart à l'équilibre de Boltzmann $\left\langle Q_{i}\right\rangle^{*}-\left\langle Q_{i}\right\rangle_{\mathrm{B}}^{*}$ soit divisé par $e . \alpha_{i}$ dépend en général de l'observable $Q_{i}$.

Remarquons que le fait de définir une probabilité $\alpha_{i}$ de désorientation par collision suppose que les propriétés de la paroi sont uniformes : par exemple, pour une cellule recouverte d'un enduit localement contaminé, ce procédé perd son sens, mais il est encore valable si une faible densité d'atomes paramagnétiques (alcalins) se répartit sur toute la paroi.

Dans le cas d'une cellule de verre nue ou recouverte d'un enduit volontairement contaminé par un miroir métallique de l'alcalin, les atomes qui heurtent la paroi ont une probabilité $\alpha \simeq 1$ de disparaître de la phase vapeur, soit par réaction chimique sur le verre, soit par condensation sur le miroir : ils sont remplacés en phase vapeur par d'autres atomes dont les spins ont en moyenne des directions correspondant à l'équilibre de Boltzmann. Nous sommes alors dans un cas particulier de collisions fortes où toutes les observables $Q$ ont la même probabilité de désorientation par collision $\alpha=\alpha_{i} \simeq 1$ et $T_{i}=\tau_{\mathrm{v}}$. Nous verrons que formellement la mise en équation s'effectue exactement comme dans le cas $\alpha_{i} \ll 1$.

3.b. Relaxation par collision sur la paroi en présence de gaz. - Par rapport au cas précédent, seul le mouvement de l'atome entre deux collisions sur la paroi change, mais nous supposons que tous les caractères de l'interaction lors d'une collision restent les mêmes. L'atome frappe la paroi après avoir diffusé dans le gaz : les processus de diffusion et de relaxation sur la paroi sont corrélés. L'effet des collisions sur la paroi se traduit par une condition aux limites de l'équation de diffusion que nous allons écrire dans le cas d'une cellule sphérique de rayon $R$, l'origine des coordonnées étant au centre de la cellule.

Par unité de temps, un certain nombre d'atomes alcalins frappent la paroi, restent adsorbés pendant le temps moyen $\tau_{\mathrm{g}}$, puis retournent en phase vapeur. On exprime ceci algébriquement à l'aide du flux sortant $J_{+}$ (nombre de particules sortant de la cellule, par unité de surface) et du flux entrant $J_{\text {.. }}$.

L'expression de ces flux est bien connue en théorie classique de la diffusion. Nous utiliserons l'expression généralisée au cas où les particules diffusantes et celles du milieu diffuseur ont une masse très différente, telle qu'on peut la déduire par exemple de [10] :

$$
\begin{aligned}
& J_{+}^{n}(R)=\bar{v}\left[\frac{n(R, t)}{4}-\frac{\lambda_{t}}{6} \frac{\partial n(r, t)}{\partial r}\right]_{(r=R)} \\
& J_{-}^{n}(R)=\bar{v}\left[\frac{n(R, t)}{4}+\frac{\lambda_{t}}{6} \frac{\partial n(r, t)}{\partial r}\right]_{(r=R)} .
\end{aligned}
$$

$\sigma^{*}(\mathbf{r}, t)$ et $Q^{*}(\mathbf{r}, t)$ obéissent à l'équation de diffusion ; nous pouvons donc par analogie avec (7) définir un flux sortant et un flux entrant $J_{+}^{\sigma^{*}}(R, t), J_{-}^{\sigma^{*}}(R, t)$ pour $\sigma^{*}(\mathbf{r}, t) ; J_{+}^{Q^{*}}(R, t), J_{-}^{Q^{*}}(R, t)$ pour $Q^{*}(\mathbf{r}, t)$.

Si on effectue à l'instant $t$ une mesure de la grandeur physique $Q^{*}$ sur les atomes qui vont frapper la paroi et sur ceux qui, après un temps de séjour $\tau_{8}$ sur la paroi, retournent en phase vapeur, le résultat est respectivement :

$$
\begin{aligned}
& J_{+}^{Q^{*}}(R, t)=\bar{v}\left[\frac{1}{4}\left\langle Q^{*}(R, t)\right\rangle-\frac{\lambda_{t}}{6}\left(\frac{\partial}{\partial r}\left\langle Q^{*}(r, t)\right\rangle\right)\right]_{(r=R)} \\
& J_{-}^{Q^{*}}(R, t)=\bar{v}\left[\frac{1}{4}\left\langle Q^{*}(R, t)\right\rangle+\frac{\lambda_{t}}{6}\left(\frac{\partial}{\partial r}\left\langle Q^{*}(r, t)\right\rangle\right)\right]_{(r=R)} .
\end{aligned}
$$

LE JOURNAL DE PHYSIQUE. - T. 28. NoB 5-6. MAT-JUIN 1967. 
Nous avons vu qu'une connaissance précise de la nature de l'interaction entre l'atome et la paroi fournit une prévision statistique de la manière dont $\sigma^{*}$ (ou $Q^{*}$ ) est affectée par une collision élémentaire. L'effet sur la matrice densité (ou sur une observable) des collisions sur la paroi peut donc être décrit par une relation entre $J_{-}^{\sigma^{*}}\left(R, t+\tau_{\mathrm{s}}\right)$ et $J_{+}^{\sigma^{*}}(R, t)$ (ou entre $J_{-}^{Q^{*}}\left(R, t+\tau_{\mathrm{s}}\right)$ et $\left.J_{+}^{Q^{*}}(R, t)\right)$.

Dans le cas d'un enduit paraffiné non contaminé, une collision produit un couplage entre les divers éléments de la matrice densité : pour la matrice densité ou une observable $Q$ quelconque, la relation cherchée est en général très complexe. Par contre, elle prend une forme simple pour les observables $Q_{i}$ que les collisions sur la paroi ne couplent à aucune autre :

$$
J_{-}^{Q_{i}^{*}}\left(R, t+\tau_{\mathrm{s}}\right)=\left(1-\alpha_{i}\right) J_{+}^{Q_{i}^{*}}(R, t) .
$$

Le temps d'adsorption $\tau_{\mathrm{s}}$ est inférieur à $10^{-9} \mathrm{~s}$, par conséquent très court devant les diverses constantes de temps d'évolution de $\sigma^{*}$. Ceci nous permet de simplifier l'équation précédente $\left({ }^{2}\right)$ et d'obtenir la condition aux limites :

$$
\left(\frac{\partial}{\partial r}\left\langle Q_{i}^{*}(r, t)\right\rangle\right)_{r=R}=-\mu_{i}\left\langle Q_{i}^{*}(R, t)\right\rangle
$$

avec

$$
\mu_{i}=3 \alpha_{i} / 2 \lambda_{t}\left(2-\alpha_{i}\right) .
$$

Le paramètre $\mu_{i}$ joue dans la suite un rôle important. Il a les dimensions de l'inverse d'une longueur : $1 / \mu_{i}$ est de l'ordre de $\lambda_{t} / \alpha_{i}$ et s'interprète comme la distance minimum que doit parcourir un atome orienté, confiné près de la paroi avant de se désorienter sur la paroi (cf. fig. 1).

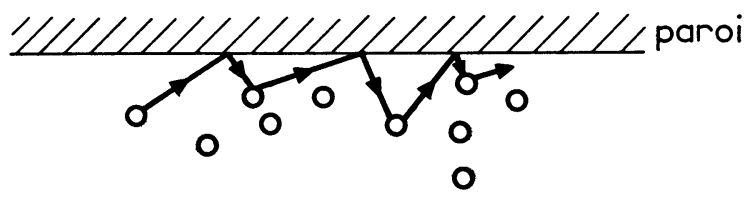

FIG. 1. - Le plus court trajet que peut parcourir un atome alcalin confiné près de la paroi avant d'avoir subi les $1 / \alpha_{i}$ collisions nécessaires pour lui faire perdre son orientation $\left\langle Q_{i}\right\rangle$, est de l'ordre de $\lambda_{\mathrm{t}} / \alpha_{i}$.

4. RelaXATION PAR GOLlisions SUR LE GAZ. - Nous faisons l'hypothèse que lors d'une collision entre un atome alcalin et un atome de gaz rare l'interaction désorientatrice est aléatoire faible et de type magnétique.

(2) Dans le cas particulier où $Q_{i}$ représente une " cohérence hyperfine ", si on s'intéresse au déplacement de fréquence produit par les collisions sur la paroi, il y a lieu d'introduire devant $1-\alpha_{i}$ (éq. 8) un facteur imaginaire du type e ${ }^{i \Delta \omega \tau_{\mathrm{s}}}$ : il traduit le fait que pendant la durée de l'adsorption l'atome est soumis, en plus de la perturbation magnétique, au hamiltonien aléatoire $\Delta a(t) \mathbf{S}$ I ( $a$ étant la constante de structure hyperfine) qui ne peut pas induire de transitions entre niveaux $F, m_{\mathrm{F}}$ différents mais cause un déplacement de fréquence, ce qui a été mis en évidence expérimentalement [12].
L'étude théorique de la relaxation provoquée par une ou plusieurs interactions du type précédent a permis de démontrer le résultat suivant : si le champ $H_{0}$ est faible (écart Zeeman $\omega_{F}$ petit devant l'écart hyperfin $\Delta w)$ quelle que soit la valeur des temps de corrélation $\tau_{\mathrm{e}}$, ce sont toujours les mêmes observables $Q_{i}$ qui relaxent avec une constante de temps unique [2]. Ces observables sont donc les mêmes dans l'hypothèse précédente pour la relaxation sur le gaz seul et pour la relaxation sur une paroi enduite de paraffine (collisions faibles de type magnétique) $\left({ }^{3}\right)$.

Pour les observables $Q_{i}$, l'équation d'évolution, sous le seul effet des collisions sur le gaz, est la suivante :

$$
\frac{\mathrm{d}}{\mathrm{d} t}\left\langle Q_{i}\right\rangle^{*}=-\frac{1}{T_{i}^{\prime}}\left\langle Q_{i}\right\rangle^{*}
$$

avec

$$
1 / T_{i}^{\prime}=N_{0} \beta_{i} \sigma \bar{v}_{\text {rel }} p / p_{0}
$$

$N_{0}$ nombre d'atomes de gaz par unité de volume à la pression $p_{0}$;

$\bar{v}_{\text {rel }}$ vitesse relative des atomes d'alcalin et de gaz rare.

$$
\bar{v}_{\mathrm{rel}}=4 \sqrt{\frac{R T}{2 \pi}\left(\frac{1}{M_{1}}+\frac{1}{M_{2}}\right)}
$$

$\sigma$ section efficace de désorientation alcalin-gaz rare.

La théorie fournit le rapport des diverses constantes de temps $T_{i}^{\prime}$. Nous posons

$$
1 / T_{i}^{\prime}=\beta_{i} / T_{1 \mathrm{~s}}^{\prime}
$$

$T_{1 \mathrm{~s}}^{\prime}$ étant le temps de relaxation longitudinale pour un spin $\mathbf{S}$ isolé (c'est-à-dire pour un isotope de l'atome alcalin considéré, qui n'aurait pas de spin nucléaire). En particulier, les observables S.I, $I_{z}, Q_{e}$ relaxent avec les constantes de temps uniques $T_{H}^{\prime}, T_{n}^{\prime}, T_{e}^{\prime}$ :

$$
\begin{aligned}
1 / T_{e}^{\prime} & =C\left[\frac{j\left(\omega_{F}\right)-j(\Delta w)}{(2 I+1)^{2}}+j(\Delta w)\right] \\
1 / T_{n}^{\prime} & =\frac{C}{(2 I+1)^{2}}\left[j\left(\omega_{F}\right)+j(\Delta w)\right] \\
1 / T_{H}^{\prime} & =C j(\Delta w)
\end{aligned}
$$

avec $j(\omega)=1 /\left(1+\omega^{2} \tau_{\mathrm{c}}^{2}\right), \quad C=1 / T_{1 S}^{\prime}, \Delta w=$ écart hyperfin.

En phase gazeuse, on s'attend à ce que la durée de la collision (de l'ordre de $10^{-12} \mathrm{~s}$ ) soit un temps de

(3) Si l'interaction désorientatrice alcalin-gaz rare n'est pas une interaction faible de type magnétique, la situation est la suivante : il existe bien encore un ensemble d'observables caractérisées par une constante de temps unique pour la relaxation sur le gaz, mais ces observables diffèrent en général des $Q_{\mathrm{i}}$, sauf peut-être un petit nombre d'entre elles $Q_{\imath}$. Pour une paroi de verre nue ou recouverte d'un miroir alcalin $\alpha=1$ quelle que soit l'observable envisagée, et la suite du calcul s'adapte directement à toutes les observables de ce nouvel ensemble. Si la paroi est recouverte d'un enduit non contaminé, la suite du calcul est seulement valable pour les observables $Q_{j}$ ou l'une de leurs combinaisons linéaires. 
corrélation. S'il n'en existe pas d'autres $\left(^{4}\right)$, puisque $\tau_{c}$ est très court, nous avons $j(\Delta w)=j\left(\omega_{F}\right)=1$. Les formules (14) se simplifient pour donner :

$$
\begin{aligned}
1 / T_{e}^{\prime} & =C \\
1 / T_{n}^{\prime} & =2 C /(2 I+1)^{2} \\
1 / T_{H}^{\prime} & =C .
\end{aligned}
$$

Nous avons donc $T_{n}^{\prime} / T_{e}^{\prime}=(2 I+1)^{2} / 2$ et $T_{e}^{\prime}=T_{H}^{\prime}$.

Notons que, d'après sa définition, $\sigma$ représente la section efficace de désorientation pour un isotope alcalin qui n'aurait pas de spin nucléaire et qui serait donc caractérisé par une constante de temps unique $T_{1 S}^{\prime}$. Ainsi définie, la section efficace est indépendante de la multiplicité de l'état fondamental et est la même pour divers isotopes. C'est ce paramètre qui a fait l'objet des études théoriques, en particulier celles de Herman [6], [7]. Par contre, les paramètres fournis par l'expérience sont $\beta_{i} \sigma=\sigma_{i}$. On voit l'importance qu'il y a à bien préciser la nature de l'observable $Q_{i}$ mesurée ainsi qu'à vérifier dans chaque cas la validité des hypothèses utilisées pour évaluer $j\left(\omega_{\mathrm{F}}\right)$ et $j(\Delta w)$ lorsqu'on veut déduire $\sigma$ de la valeur $\sigma_{i}$ mesurée.

5. Équation D'ÉVOlution GLOBALE D'UNE OBSERVABLE. - L'évolution globale a la forme suivante :

$$
\frac{\mathrm{d}\langle Q\rangle}{\mathrm{d} t}=\frac{\mathrm{d}^{(\mathbf{1})}}{\mathrm{d} t}\langle Q\rangle+\frac{\mathrm{d}^{(2,3)}}{\mathrm{d} t}\langle Q\rangle+\frac{\mathrm{d}^{(\mathbf{4})}}{\mathrm{d} t}\langle Q\rangle .
$$

Le premier terme traduit l'évolution de $\langle Q\rangle$ sous l'effet du hamiltonien statique $\mathscr{H}_{0}$. Il s'écrit :

$$
\frac{\mathrm{d}^{(\mathbf{1})}\langle Q\rangle}{\mathrm{d} t}=-i \operatorname{Tr}\left\{Q\left[\mathscr{H}_{0}, \sigma\right]\right\} \text {. }
$$

Nous avons vu qu'on l'élimine en passant en représentation d'interaction

$$
\frac{\mathrm{d}\langle Q\rangle^{*}}{\mathrm{~d} t}=\frac{\mathrm{d}^{(2,3)}}{\mathrm{d} t}\langle Q\rangle^{*}+\frac{\mathrm{d}^{(4)}}{\mathrm{d} t}\langle Q\rangle^{*} .
$$

Le deuxième terme traduit l'effet de la relaxation par collisions sur la paroi après diffusion dans le gaz (processus 2 et 3 corrélés), le troisième terme traduit l'effet de la relaxation par collisions sur le gaz (processus 4). Les processus 2 et 3 d'une part, 4 d'autre part ne sont pas corrélés. Leur étude menée de manière indépendante a abouti à un résultat particulièrement simple en ce qui concerne les observables $Q_{i}^{*}$, on a :

$\frac{\mathrm{d}}{\mathrm{d} t}\left\langle Q_{i}^{*}(\mathbf{r}, t)\right\rangle=D \nabla^{2}\left\langle Q_{i}^{*}(\mathbf{r}, t)\right\rangle-\frac{1}{T_{i}^{\prime}}\left\langle Q_{i}^{*}(\mathbf{r}, t)\right\rangle$

avec la condition aux limites :

$$
\left[\frac{\partial}{\partial r}\left\langle Q_{i}^{*}(\mathbf{r}, t)\right\rangle\right]_{r=R}=-\mu_{i}\left\langle Q_{i}^{*}(R, t)\right\rangle .
$$

(4) Des mesures de relaxation récentes effectuées sur $\mathrm{Rb}$ en présence de krypton [13] ont montré que l'interaction désorientatrice en phase gazeuse était dans ce cas caractérisée par l'existence d'un second temps de corrélation de l'ordre de $10^{-8} \mathrm{~s}$.
En particulier dans le cas d'une grandeur longitudinale, le terme $\mathrm{d}^{(\mathbf{1})} Q / \mathrm{d} t$ est nul $\left(\langle Q\rangle=\langle Q\rangle^{*}\right)$ et l'opérateur $Q$ s'exprimant de manière unique en fonction des $Q_{i}$, l'évolution globale de $\langle Q\rangle$ peut se calculer complètement et de manière exacte à partir des $4 I+1$ équations analogues à (16) et (17).

6. Choix des conditions initiales. - Pour décrire totalement l'évolution du système, il faut en outre connaître son état à l'instant initial à partir duquel on étudie la relaxation. En pratique, c'est l'état d'équilibre que le pompage optique permet d'atteindre en présence des différents processus de relaxation analysés ci-dessus : $\left\langle Q_{i}^{*}(\mathbf{r}, t=0)\right\rangle$ est donc la solution stationnaire des équations (16) et (17) lorsqu'on ajoute dans le second membre de $(16)$ le terme $\frac{\mathrm{d}^{(5)}}{\mathrm{d} t}\left\langle Q_{i}^{*}(\mathbf{r}, t)\right\rangle$ qui décrit l'évolution de $Q_{i}^{*}$ sous le seul effet du pompage optique.

Dans les cas de pompage usuels, $\mathrm{d}^{(5)}\langle Q\rangle / \mathrm{d} t$ a généralement une forme très complexe pour les observables $Q_{i}^{*}$, que les absorptions et réémissions successives de photons couplent entre elles. Dans un but de simplification, nous envisagerons ici seulement un procédé de pompage tel que pour une ou plusieurs observables $Q_{i}^{*}(\mathbf{r}, t)$ on puisse écrire l'équation d'évolution sous la forme simple suivante :

$$
\frac{\mathrm{d}^{(\mathbf{5})}}{\mathrm{d} t}\left\langle Q_{i}^{*}(\mathbf{r}, t)\right\rangle=\frac{q_{i}^{* 0}-\left\langle Q_{i}^{*}(\mathbf{r}, t)\right\rangle}{k_{i} T_{p}(\mathbf{r})} .
$$

Notons que ce type d'équation est celui que l'on obtient rigoureusement par exemple dans le cas de $\left\langle S_{z}\right\rangle$ et d'un alcalin sans spin nucléaire. $q_{i}^{0}$ est la valeur maximum que le type de pompage utilisé permet d'obtenir pour la valeur moyenne locale de l'observable $Q_{i}^{*} . k_{i}$ est un coefficient numérique qui se calcule explicitement, connaissant les caractéristiques du pompage. $T_{p}(\mathbf{r})$ est le temps moyen qui séparerait l'absorption successive de 2 photons par un atome supposé figé au point $\mathbf{r}$. Ce facteur fait intervenir la répartition géométrique de l'intensité lumineuse pompante dans le volume $V: T_{p}(\mathbf{r})$ est inversement proportionnel à $I_{p}(\mathbf{r})$.

Nous ne traiterons explicitement le calcul que dans le cas où $I_{p}(\mathbf{r})$ est une fonction uniforme dans le volume $V$; nous supposons également que $q_{i}^{* 0}$ et $k_{i}$ ne dépendent pas de $\mathbf{r}$, c'est-à-dire que l'intensité pompante a même polarisation et même forme spectrale en tous les points du volume $V$. Ces hypothèses sont raisonnables lorsque l'absorption par la vapeur alcaline est très faible. $\left\langle Q_{i}(\mathbf{r}, t=0)\right\rangle$ satisfait alors les équations :

$$
\begin{aligned}
& D \nabla^{2}\left\langle Q_{i}^{*}(\mathbf{r}, t=0)\right\rangle-\frac{1}{T_{i}^{\prime}}\left\langle Q_{i}^{*}(\mathbf{r}, t=0)\right\rangle \\
& \quad+\frac{q_{i}^{* 0}-\left\langle Q_{i}^{*}(\mathbf{r}, t=0)\right\rangle}{k_{i} T_{p}}=0 \\
& {\left[\frac{\partial}{\partial r}\left\langle Q_{i}^{*}(\mathbf{r}, t=0)\right\rangle\right]_{r=R}=-\mu_{i}\left\langle Q_{i}^{*}(R, t=0)\right\rangle .}
\end{aligned}
$$


Ces équations sont indépendantes de $\theta$ et de $\varphi$ et par conséquent $Q_{i}^{*}(\mathbf{r}, t=0)$ est isotrope.

Nous discutons maintenant la validité de l'équation (18).

Lorsque le pompage optique peut être considéré comme apportant une petite perturbation aux effets dus à la relaxation, on obtient avec une très bonne approximation l'évolution globale des observables $Q_{i}^{*}$ en utilisant (18) pour décrire l'effet du pompage (la démonstration est analogue à celle que l'on trouve dans la référence ([15], § III.D).

$\mathrm{Au}$ contraire, lorsque le pompage en lumière intense crée à l'équilibre un état très différent de celui de Boltzmann, et dépendant très peu des effets de la relaxation, $\mathrm{d}^{(\mathbf{5})}\langle Q\rangle / \mathrm{d} t$ est le seul terme important pour décrire l'évolution globale du système et son état d'équilibre. On peut montrer qu'il existe un ensemble $Q_{p}$ d'observables pour lesquelles il est exact d'écrire ce terme sous la forme (18), mais en général les observables $Q_{i}$ sont différentes des $Q_{p}$, et s'expriment de manière unique comme une combinaison linéaire de plusieurs d'entre elles. A l'équilibre, on obtient :

$$
\left\langle Q_{p}^{*}(\mathbf{r}, t=0)\right\rangle=q_{p}^{* 0} .
$$

Ceci signifie que la répartition dans le volume $V$ de la valeur moyenne locale des observables $Q_{p}^{*}$, et par suite $Q_{i}^{*}$, ne dépend pas de $\mathbf{r}$. Remarquons que le même résultat aurait été obtenu si nous avions écrit que $Q_{i}^{*}$ satisfaisait (18).

En conclusion, les équations (19) et (20) ne sont qu'exceptionnellement valables (procédé de pompage particulier, observables particulières), mais elles donnent des résultats corrects sur la distribution dans le volume obtenue pour toutes les observables $Q_{i}^{*}$ aux très faibles et aux très fortes intensités pompantes. C'est en fait dans ces cas qu'il est intéressant de connaître les résultats du calcul.

Remarquons que nos hypothèses diffèrent de celles adoptées par P. Minguzzi et al. [14] en deux points. Ces auteurs adoptent pour $\mathrm{d}^{(5)}\langle Q\rangle / \mathrm{d} t$ l'expression suivante :

$$
\frac{\mathrm{d}^{(5)}\left\langle Q_{i}(\mathbf{r}, t)\right\rangle}{\mathrm{d} t}=I_{p}(\mathbf{r}) .
$$

Ils envisagent le cas où la tension de vapeur d'alcalin dans la cellule est assez forte pour produire une absorption considérable du faisceau pompant $F_{p}$ entre la face d'entrée et la face de sortie, et par suite une baisse exponentielle de $I_{p}$ suivant l'axe du faisceau pompant. Nous ne nous intéressons pas ici à un effet de ce type, car les mesures que nous avons effectuées sont faites à une faible tension de vapeur.

D'autre part, puisque le second membre de (21) ne dépend pas de $\left\langle Q_{i}^{*}(\mathbf{r}, t)\right\rangle$, ils admettent, implicitement, qu'en tous les points du volume $V, Q_{i}^{*}(\mathbf{r}, t)$ est très éloigné de la valeur maximum $q_{i}^{* 0}$.

III. Principales étapes du calcul. - Au § II, nous sommes arrivées à la conclusion que la valeur moyenne locale de l'observable $Q_{i}^{*}$ était une solution de :

$$
\frac{\partial y(\mathbf{r}, t)}{\partial t}=D \nabla^{2} y(\mathbf{r}, t)-\frac{1}{T_{i}^{\prime}} y(\mathbf{r}, t)
$$

satisfaisant à la condition aux limites :

$$
\left[\frac{\partial y(\mathbf{r}, t)}{\partial r}\right]_{\mathrm{r}=\mathrm{R}}=-\mu_{i} y(R, t)
$$

et à une condition initiale que nous supposons isotrope.

La résolution d'un problème de ce type est classique (voir par exemple [16]), aussi nous n'en indiquons que les étapes essentielles, pour une discussion plus détaillée nous renvoyons à [17].

1. SolUtions PARTIGULIÈres ET SOLUTION GÉNÉRALE. - La recherche des solutions du type

$$
y=u(r, \theta, \varphi) v(t)
$$

ayant un sens physique conduit au résultat :

$$
\begin{gathered}
v(t)=v_{0} \mathrm{e}^{-\left(D \omega^{2}+1 / T_{1}^{\prime}\right) t} \\
\nabla^{2} u+\omega^{2} u=0 .
\end{gathered}
$$

On est ramené à résoudre l'équation (25) avec la condition aux limites (23). Ce problème admet pour solutions les fonctions

$$
y_{v}^{l m}=Y_{l m}(\theta, \varphi) \frac{J_{l+1 / 2}(\omega r)}{\sqrt{\omega r}}
$$

où $Y_{l m}(\theta, \varphi)$ sont les harmoniques sphériques d'ordre $l$ et de rang $m, J_{l+1 / 2}(x)$ les fonctions de Bessel d'ordre $l+1 / 2$ et où $\omega=\omega_{i}^{\mathrm{v}, l}$ fait partie de la suite discrète des racines de l'équation aux valeurs propres constituée par l'équation aux limites (équation 23).

Nous posons :

$$
1 / \tau_{i}^{\nu, l}=D\left(\omega_{i}^{\nu, l}\right)^{2}+1 / T_{i}^{\prime} .
$$

On peut démontrer que les fonctions $y_{v}^{l m} \mathrm{e}^{-t / \tau_{i}^{\nu, l}}$ sont deux à deux orthogonales ; en outre, elles forment un système complet dans l'ensemble des fonctions réelles continues définies sur le segment $[\mathrm{O}, \mathrm{R}]$, solutions de notre problème. La solution générale est donc de la forme :

$$
y(r, \theta, \varphi, t)=\sum_{v, l, m} A_{i}^{v, l, m} Y_{l, m}(\theta, \varphi) \frac{J_{l+1 / 2}\left(\omega_{i}^{v, l} r\right)}{\sqrt{\omega_{i}^{v, l} r}} \mathrm{e}^{-t / \tau_{i}^{v, l}}
$$


Les coefficients $A_{i}^{v, l, m}$ se déduisent de la connaissance de la condition initiale

$$
y(r, \theta, \varphi, t=0)=\left\langle Q_{i}^{*}(\mathbf{r}, t=0)\right\rangle .
$$

2. Conséquence de L'isotropie de la GOndition INITIALE. - Si à l'instant $t=0$ nous supposons une répartition spatiale isotrope pour la valeur moyenne locale de l'observable $Q_{i}^{*}$ à l'intérieur de la cellule, il en résulte que

$$
A_{i}^{v, l, m}=0 \quad \text { quel que soit } l \neq 0 .
$$

Donc, par la suite, n'interviendront que les solutions de l'équation aux valeurs propres écrite pour $l=0$, soit $\omega_{i}^{v, 0}=\omega_{i}^{(v)}$, et la partie radiale de la solution sera de la forme $\sin \omega_{i}^{(\nu)} r / \omega_{i}^{(\nu)} r$. L'équation (28) se simplifie :

$$
y(r, t)=\sum_{\nu} A_{i}^{(\nu)} \frac{\sin \omega_{i}^{(\nu)} r}{\omega_{i}^{(v)} r} \mathrm{e}^{-t / \tau_{i}^{(\nu)}}
$$

avec

$$
1 / \tau_{i}^{(\nu)}=D\left(\omega_{i}^{(\nu)}\right)^{2}+1 / T_{i}^{\prime}
$$

3. RÉSOLUTION DE L'ÉQUATION AUX VALEURS PROPRES. - Pour $l=0$, l'équation aux limites s'écrit :

$$
\operatorname{tg} \omega R=\omega R /\left(1-\mu_{i} R\right) .
$$

Cette équation a une infinité dénombrable de solutions positives $h_{v}=\omega_{i}^{(v)} R>0 . \quad h_{v}(\nu$ entier $)$ est l'abscisse du vième point d'intersection des courbes représentant les fonctions :

$$
y_{1}=\operatorname{tg} h \quad y_{2}=h /\left(1-\mu_{i} R\right) .
$$

La figure 2 montre que $(\nu-1) \pi<h_{\nu}<\nu \pi$.

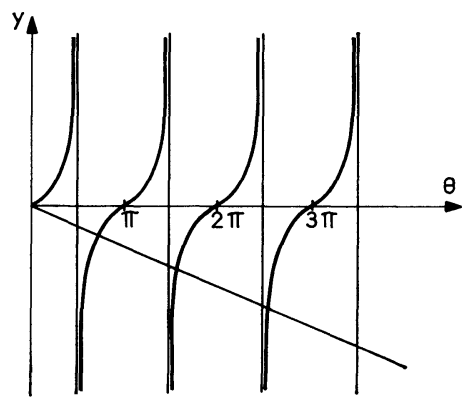

FIG. 2. - Les valeurs propres $h_{\mathrm{y}}=\omega_{i}^{(\mathrm{v})} R$ sont les abscisses des points d'intersection de la courbe représentant $y=\operatorname{tg} \theta$ et de la droite $y=\theta /\left(1-\mu_{i} R\right)$.

La valeur de la constante de temps $\tau_{i}^{(v)}$ se déduit de $h_{\nu}$ en utilisant l'équation (30). Nous posons :

$$
\begin{aligned}
1 / \tau_{i}^{(v)} & =1 / T_{i}^{(v)}+1 / T_{i}^{\prime} \\
1 / T_{i}^{(\nu)} & =D\left(h_{v}\right)^{2} / R^{2} .
\end{aligned}
$$

Chacune des constantes de temps $T_{i}^{(v)}$ est associée à un mode $\nu$ de diffusion vers la paroi.
La résolution de l'équation aux valeurs propres est intéressante dans les cas limites $\mu_{i} R \gg 1$ et $\mu_{i} R \ll 1$.

a) Limite aux pressions fortes. - Lorsque la condition $\mu_{i} R \gg 1$ est satisfaite, les solutions de l'équation aux valeurs propres tendent vers $v \pi$. Un développement limité en $1 / \mu_{i} R$ au voisinage de cette valeur permet d'obtenir :

$$
\frac{1}{\tau_{i}^{(v)}}=\frac{D}{R^{2}} \nu^{2} \pi^{2}\left(1-\frac{2}{\mu_{i} R}\right)+\frac{1}{T_{i}^{\prime}} .
$$

Aux très fortes pressions, les temps de relaxation deviennent indépendants de la qualité de la paroi; ce fait est interprété physiquement au $\S$ IV.A.1.

b) Limite aux faibles pressions pour un bon enduit. Nous cherchons la limite de $\omega_{i}^{(v)} R$ dans le cas d'une pression faible $\left(\mu_{i} R \ll 1\right)$ mais suffisamment grande pour que l'équation de diffusion ait encore un sens $\lambda_{t} \ll R$. (On vérifie aisément que la compatibilité de ces deux conditions exige un bon enduit $\alpha_{i} \ll 1$.)

En ce qui concerne le premier mode, un développement limité de l'équation aux valeurs propres au voisinage de $h=0$ et de $\mu_{i} R=0$ permet d'obtenir : $\left(h_{1}\right)^{2}=3 \mu_{i} R$.

La valeur limite $\tau_{i}^{0}$ vers laquelle tend $T_{i}^{(1)}$ se calcule donc en utilisant les relations (32), (10) et (3) :

$$
\frac{1}{\tau_{i}^{0}}=\frac{3 \mu_{i} D}{R}=\frac{3 \alpha_{i} \bar{v}}{2\left(2-\alpha_{i}\right) R} \approx \frac{3 \alpha_{i} \bar{v}}{4 R} .
$$

Il est intéressant de comparer $\tau_{i}^{0}$ au temps de relaxation caractérisant $Q_{i}^{*}$ en l'absence de gaz : $T_{i}=\tau_{v} / \alpha_{i}$. Un calcul simple $\left(^{5}\right)$ permet d'évaluer $\tau_{v}=4 R / 3 \bar{v}$ et montre donc que $\tau_{i}^{0}=T_{i}$.

Pour les modes d'ordre supérieur, on vérifie qu'aux faibles pressions $h_{v}$ vaut pratiquement $(\nu-1) \pi$, et par conséquent les constantes de temps $T_{i}^{(v)}$ proportionnelles à $p$ deviennent très courtes, d'autant plus que le mode $\vee$ est plus élevé, mais nous verrons ( $\$$ III.4) que simultanément leur poids tend vers zéro.

c) Dans toute la zone des pressions où l'équation de diffusion est valable, les valeurs de $h_{\nu}$ sont données en fonction de $\mu_{i} R$ dans le tableau I pour $\nu=1$ et $\nu=2$. Dans le même tableau figure en fonction de $\mu_{2} R$ le pouvoir tampon du gaz étranger

$$
T_{i}^{(1)} / T_{i}=3 \mu_{i} R /\left(h_{1}\right)^{2} .
$$

(5) Les atomes sont confinés dans le volume $\mathrm{V}$ délimité par la paroi de surface $S$. Le nombre d'atomes frappant la paroi par $\mathrm{cm}^{2}$ et par seconde est $\frac{N}{V} \frac{\bar{v}}{4}$. Le nombre total de collisions par unité de temps sur la paroi est $N \frac{S}{V} \frac{\bar{v}}{4}$ qui, pour une cellule sphérique, donne $N 3 \bar{v} / 4 R=N / \tau_{\mathrm{v}}$. 


\section{TABLEAU I}

$\begin{array}{cccc} & & 3 \mu_{\mathrm{i}} R & \\ h_{1} & \mu_{\mathrm{i}} R & \frac{\left.h_{1}\right)^{2}}{2} & h_{2} \\ - & - & - & - \\ 0,09 & 0,002 & 1,002 & 4,48 \\ 0,26 & 0,023 & 1,005 & 4,49 \\ 0,52 & 0,093 & 1,018 & 4,51 \\ 0,78 & 0,214 & 1,042 & 4,54 \\ 1,04 & 0,39 & 1,082 & 4,57 \\ 1,22 & 0,55 & 1,12 & 4,61 \\ 1,48 & 0,87 & 1,19 & 4,68 \\ 1,66 & 1,14 & 1,28 & 4,74 \\ 1,83 & 1,49 & 1,33 & 4,81 \\ 2,00 & 1,94 & 1,45 & 4,90 \\ 2,35 & 3,35 & 1,82 & 5,14 \\ 2,53 & 4,61 & 2,17 & 5,30 \\ 2,70 & 6,80 & 2,78 & 5,59 \\ 2,96 & 17,8 & 6,25 & 5,91 \\ 3,04 & 29,9 & 10 & 6,1 \\ 3,07 & 44,8 & 14,3 & 6,1 \\ 3,11 & 89,9 & 33 & 6,19 \\ 3,14 & \infty & 0 & 6,28\end{array}$

Les valeurs tabulées permettent d'évaluer

et

$$
T_{i}^{(1)}=T_{i} \cdot \frac{3 \mu_{\mathrm{i}} R}{\left(h_{1}\right)^{2}}
$$

$$
T_{i}^{(2)}=T_{i}^{(1)} \cdot\left(\frac{h_{1}}{h_{2}}\right)^{2}
$$

4. Importance relative des différents modes. - La condition initiale à satisfaire (19) est :

$D \nabla^{2} y(\mathbf{r}, t=0)-\frac{1}{T_{i}^{\prime}} y(\mathbf{r}, t=0)+\frac{q_{i}^{* 0}-y(\mathbf{r}, t=0)}{k_{i} T_{p}}=0$ mais nous savons que, quel que soit $t$ :

$$
D \nabla^{2} y(\mathbf{r}, t)-\frac{1}{T_{i}^{\prime}} y(\mathbf{r}, t)=\frac{\partial}{\partial t} y(\mathbf{r}, t) .
$$

Tenant compte de (29) et (35), on peut réécrire la condition initiale :

$$
\sum_{\nu} A_{i}^{(\nu)}\left(\frac{1}{\tau_{i}^{(\nu)}}+\frac{1}{k_{i} T_{p}}\right) \frac{\sin \omega_{i}^{(\nu)} r}{\omega_{i}^{(v)} r}=\frac{q_{i}^{* 0}}{k_{i} T_{p}} .
$$

Nous avons vu que le système des fonctions $\frac{\sin \omega_{i}^{(\nu)} r}{\omega_{i}^{(v)} r}$ forme un système orthogonal complet dans l'espace des solutions du problème physique. On peut montrer (cf. [16], § VI.3) que, bien que le second membre de (36) ne satisfasse pas nécessairement à la condition aux limites (23), il admet cependant un développement convergent en tout point à l'intérieur du segment $[\mathrm{O}, \mathrm{R}[$, de la forme :

$$
\frac{q_{i}^{* 0}}{k_{i} T_{p}}=\sum_{\nu} \frac{K_{i}^{(v)}}{k_{i} T_{p}} \frac{\sin \omega_{i}^{(v)} r}{\omega_{i}^{(v)} r}
$$

avec :

$$
K_{i}^{(\nu)}=2 q_{i}^{*} \frac{\left(\sin h_{v} / h_{v}\right)-\cos h_{v}}{1-\left(\sin 2 h_{v} / 2 h_{v}\right)}
$$

on obtient alors :

$$
A_{i}^{(v)}=K_{i}^{(v)} \frac{1 / k_{i} T_{p}}{1 / \tau_{i}^{(v)}+1 / k_{i} T_{p}} .
$$

La mesure physique porte non pas sur la valeur moyenne locale de l'observable $Q_{i}^{*}$ mais sur sa valeur moyenne mesurée sur la totalité du volume $V_{d}$ de la cellule que traverse le faisceau détecteur :

$$
\left\langle Q_{i}^{*}(t)\right\rangle=\frac{1}{V_{d}} \int_{V_{d}} \mathrm{~d}^{3} \mathbf{r}\left\langle Q_{i}^{*}(\mathbf{r}, t)\right\rangle .
$$

Nous définissons les poids $\gamma_{i}^{(v)}$ des constantes de temps $\tau_{i}^{(v)}$ caractérisant l'évolution de $\left\langle Q_{i}^{*}\right\rangle$ à partir de sa valeur initiale $\left\langle Q_{i}^{*}\right\rangle_{0}$ par l'équation :

$$
\left\langle Q_{i}^{*}\right\rangle=\left\langle Q_{i}^{*}\right\rangle_{0} \sum_{\nu} \gamma_{i}^{(v)} \mathrm{e}^{-t / \tau_{i}^{(v)}} .
$$

Lorsque la détection porte sur la totalité du volume $V$, on calcule aisément que $\gamma_{i}^{(v)}$ se déduit de $A_{i}^{(v)}$ par la relation suivante :

$$
\gamma_{i}^{(v)}=\frac{3 A_{i}^{(v)}}{\left\langle Q_{i}\right\rangle_{0}} \cdot \frac{1}{h_{\nu}^{2}}\left[\frac{\sin h_{\nu}}{h_{\nu}}-\cos h_{\nu}\right]
$$

ou, tenant compte de (37) et (38) :

$$
\gamma_{i}^{(v)}=\frac{6 q_{i}^{* 0}}{\left\langle Q_{i}\right\rangle_{0}} \cdot \frac{1}{h_{v}^{2}} \cdot \frac{\left[\left(\sin h_{v} / h_{v}\right)-\cos h_{v}\right]^{2}}{1-\frac{\sin 2 h_{v}}{2 h_{v}}} \cdot \frac{1 / k_{i} T_{p}}{1 / \tau_{i}^{(v)}+1 / k_{i} T_{p}} .
$$

Cette expression conduit à des résultats simples dans les cas extrêmes $\mu_{i} R \gg 1$ et $\mu_{i} R \ll 1$.

Cas $\mu_{i} R \gg 1: h_{\nu} \approx \nu \pi$ et l'on déduit de $(40):$

$$
\frac{\gamma_{i}^{(v)}}{\gamma_{i}^{(1)}}=\frac{1}{v^{2}} \cdot \frac{1 / \tau_{i}^{(1)}+1 / k_{i} T_{p}}{1 / \tau_{i}^{(v)}+1 / k_{i} T_{p}}
$$

On trouvera au § IV.A une discussion détaillée de ce résultat.
Cas $\mu_{i} R \ll 1$ : Pour le premier mode $\left(h_{1} \rightarrow 0\right)$, la valeur limite de $\gamma_{i}^{(1)}$ est calculée à partir de l'équation $(40)$ :

$$
\gamma_{i}^{(1)}=\frac{q_{i}^{* 0}}{\left\langle Q_{i}\right\rangle_{0}} \cdot \frac{1 / k_{i} T_{p}}{1 / \tau_{i}^{(1)}+1 / k_{i} T_{p}} .
$$

Pour les modes $\nu>1, h_{\nu} \approx(\nu-1) \pi$, l'équation $(40)$ montre que 


$$
\frac{\gamma_{i}^{(\nu)}}{\gamma_{i}^{(1)}}=\frac{6}{(\nu-1)^{2} \pi^{2}}\left(\mu_{i} R\right)^{2} .
$$

Donc, en pratique, seul le premier mode subsiste, dans ce cas.

\section{RESULTATS DU CALCUL THEORIQUE ET INTERPRETATION}

Nous résumons ci-après les principaux résultats relatifs à l'évolution dans le temps de la valeur moyenne d'une observable $Q$, lorsque la relaxation a pour cause les deux processus envisagés :

a) Collisions sur la paroi après diffusion dans le gaz;

b) Collisions sur les atomes du gaz tampon.

L'évolution de $Q$ se déduit de celle des observables $Q_{i}$ que nous avons choisies pour base (cf. $\S$ II.3.a, équation (5)).

Une observable $Q_{i}$ relaxe avec une infinité dénombrable de constantes de temps $\tau_{i}^{(v)}$, chacune étant associée à un mode de diffusion $\nu$ vers la paroi. Le poids $\gamma_{i}^{(v)}$ de chaque mode dépend de la répartition géométrique de la grandeur physique associée à l'observable $Q_{i}$ à l'instant initial à partir duquel on étudie la relaxation :

avec

$$
\left\langle Q_{i}(t)\right\rangle=\left\langle Q_{i}\right\rangle_{0} \sum_{\nu} \gamma_{i}^{(\nu)} \mathrm{e}^{-t / \tau_{i}^{(\nu)}}
$$

$$
1 / \tau_{i}^{(\nu)}=1 / T_{i}^{(\nu)}+1 / T_{i}^{\prime}
$$

$1 / T_{i}^{(v)}$ traduit l'effet de la relaxation sur la paroi pour l'observable $Q_{i}$, après diffusion à travers le gaz selon le $\nu^{\text {ième }}$ mode.

$1 / T_{i}^{\prime}$ traduit l'effet de la relaxation sur le gaz pour l'observable $Q_{i}$.

Dans les expressions de $T_{i}^{(v)}$ et $\gamma_{i}^{(v)}$ intervient le paramètre $\mu_{i}$ sous la forme du produit sans dimension $\mu_{i} R$. Nous rappelons que $1 / \mu_{i}$ représente la distance minimum que doit parcourir un atome orienté confiné près de la paroi avant de se désorienter sur elle. On remarque que les résultats prennent une forme totalement différente suivant que $\mu_{i} R$ est petit ou grand devant 1 . La raison en est la suivante : si la paroi est peu désorientante et la pression de gaz faible $\mu_{i} R \ll 1$, un atome peut traverser plusieurs fois la cellule avant de se désorienter par le processus $a$. Par contre, si la paroi est très désorientante ou si la pression du gaz est très forte, un atome orienté qui diffuse du centre au bord de la cellule perd son orientation avant d'avoir une chance de s'éloigner de nouveau de la paroi $\left(1 / \mu_{i} \ll R\right)$. Dans le premier cas, on s'attend à ce que le mouvement des atomes rende uniforme $\left\langle Q_{i}(\mathbf{r}, t)\right\rangle$ dans tout le volume occupé par la vapeur, et cela même si les conditions de pompage sont différentes d'un point à un autre. Dans le second cas au contraire on s'attend à ce que l'orientation soit faible près de la paroi, et reste plus ou moins confinée dans la zone où elle est produite. Par la suite, nous ferons encore souvent allusion à ces deux cas extrêmes :

$$
\begin{array}{ll}
1 / \mu_{i} \gg R & \text { orientation " non confinée " } \\
1 / \mu_{i} \ll R & \text { orientation « confinée ». }
\end{array}
$$

On peut remarquer que l'effet de « confinement » de l'observable $Q_{i}$ est aggravé par la relaxation sur le gaz.

Comme nous l'avons vu, la théorie de la diffusion n'étant valable que pour $\lambda_{t} \ll R$, il en résulte que la condition $\mu_{i} R \ll 1$ (orientation « non confinée ») n'est réalisable qu'avec de bons enduits.

Nous analysons maintenant les expressions de $\tau_{i}^{(v)}$ et $\gamma_{i}^{(v)}$ obtenues lorsque le processus $a$ est seul présent. La même étude est faite ensuite dans le cas où les processus $a$ et $b$ agissent simultanément.

A. Résultats concernant la relaxation sous l'effet du processus $a$ seul. Etude de $T_{i}^{(\nu)}$ et $\gamma_{i}^{(\nu)}$. - En fonction de $\mu_{i} R$, du coefficient de diffusion $D_{0}$ de l'alcalin dans le gaz à la pression $p_{0}$ et du rayon $R$ de la cellule, nous avons obtenu que $T_{i}^{(v)}$ à la pression $p$ est donné par l'expression :

$$
\frac{1}{T_{i}^{(v)}}=D_{0} \frac{p_{0}}{p} \frac{h_{v}^{2}}{R^{2}}
$$

où $h_{v}$, solution de l'équation (31) comprise entre $(\nu-1) \pi$ et $\nu \pi$, est donné dans le tableau I pour $\nu=1$ et $\nu=2$. Interprétons les résultats auxquels cette expression conduit dans les cas limites :

1. GAS D'Une « ORIENTATION GONFinéE 》 $\mu_{\cdot i} R \gg 1$. Alors $h_{\nu}=v \pi$. On a :

$$
\frac{1}{T_{i}^{(v)}}=D_{0} \frac{p_{0}}{p} \frac{\nu^{2} \pi^{2}}{R^{2}}
$$

On remarque que $T_{i}^{(v)}$ est indépendant de $i$ (on obtient la même constante de temps pour toutes les observables $Q_{i}$ ) et indépendant de la qualité de l'enduit. Ce résultat s'explique de la manière suivante : un atome au centre de la cellule doit diffuser du centre au bord avant de subir une première collision sur la paroi. Ensuite, il peut diffuser par exemple dans une direction normale à celle-ci sur une distance de l'ordre de $R$ et échapper à son influence. Il peut aussi diffuser dans une direction parallèle, auquel cas, dès qu'il a parcouru une distance supérieure ou égale à $1 / \mu_{i}$, il a une chance appréciable d'effectuer les $1 / \alpha_{i}$ collisions contre la paroi qui lui font perdre son orientation. Puisque nous sommes dans des circonstances où $1 / \mu_{i}$ est petit devant $R$, le second processus est beaucoup plus probable que le premier : l'atome est emprisonné près de la paroi assez longtemps pour perdre son orientation. Le temps de relaxation est alors pratiquement le temps que met un atome initialement au centre de la cellule pour diffuser jusqu'au voisinage de la paroi : il ne dépend plus des qualités de cette dernière. 
La condition $\mu_{i} R \gg 1$ est toujours vérifiée pour une paroi totalement désorientante $\left(\alpha_{i}=1\right)$. Dans tout le domaine de pression où l'équation de diffusion est valable $\left(\lambda_{t} \ll R\right), 1 / T_{i}^{(1)}=1 / T^{(1)}$ est alors représenté par une hyperbole ( fig. 3 , courbe $a$ ). Notons que

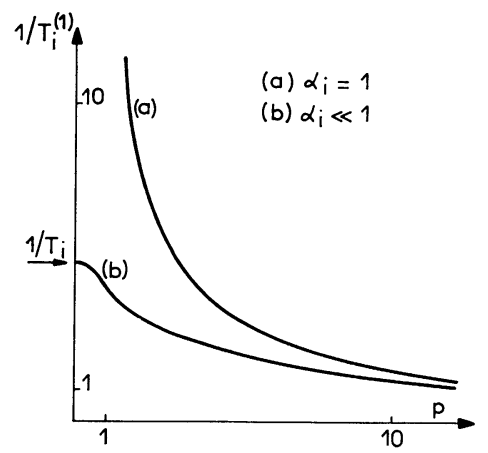

FIG. 3. - Variations de $1 / T_{i}^{(1)}$ en fonction de la pression dans le cas d'une paroi de verre nu (a) ou recouverte d'un enduit $(b)$. Les unités sont choisies de manière arbitraire.

meilleure est une paroi, plus grande est la pression nécessaire pour que la condition d'orientation « confinée " soit satisfaite. On s'attend donc à ce que les courbes donnant $1 / T_{i}^{(1)}$ en fonction de $p$ se raccordent à l'hyperbole (a) pour des pressions d'autant plus fortes que $\alpha_{i}$ est petit.

Pour une " orientation confinée », les poids $\gamma_{i}^{(v)}$ sont aussi indépendants de $i$ et de la qualité de l'enduit; ils dépendent seulement de la répartition dans le volume de la cellule de la valeur moyenne locale de l'observable $Q_{i}^{*}$ à l'instant initial. Ils en dépendent de manière assez critique comme on peut s'en apercevoir sur l'équation (41):

$$
\frac{\gamma_{i}^{(\nu)}}{\gamma_{i}^{(1)}}=\frac{1}{\nu^{2}} \cdot \frac{1 / \tau_{i}^{(1)}+1 / k_{i} T_{p}}{1 / \tau_{i}^{(\nu)}+1 / k_{i} T_{p}}
$$

Deux cas sont à envisager suivant que le système est préparé dans l'état initial avec une intensité pompante forte ou faible.

a) Intensité pompante forte :

$\mathrm{Si}$

$$
1 / k_{i} T_{p} \gg 1 / \tau_{i}^{(1)}, 1 / \tau_{i}^{(v)}
$$

nous obtenons

$$
\gamma_{i}^{(v)} / \gamma_{i}^{(1)}=1 / v^{2}
$$

Lorsque l'intensité pompante est forte, nous avons vu que la valeur moyenne locale de l'observable $Q_{i}^{*}$ est uniforme dans tout le volume $V$ à l'instant $t=0$, et égale à la valeur maximum $q_{i}^{* 0}$ compatible avec les conditions de pompage utilisées. L'équation (44) montre que le poids relatif du mode d'ordre $\nu$ décroît comme $v^{2}$. b) Intensité pompante faible :

$\mathrm{Si}$

$$
1 / k_{i} T_{p} \ll 1 / \tau_{i}^{(1)}, 1 / \tau_{i}^{(v)}
$$

nous obtenons

$$
\frac{\gamma_{i}^{(\nu)}}{\gamma_{i}^{(1)}}=\frac{1}{\nu^{2}} \cdot \frac{\tau_{i}^{(\nu)}}{\tau_{i}^{(1)}}=\frac{1}{\nu^{4}} .
$$

La condition (46) signifie que l'intensité pompante est si faible qu'en tous les points du volume $V$ on s'écarte peu de l'équilibre de Boltzmann :

$$
\left\langle Q_{i}^{*}(\mathbf{r}, t=0)\right\rangle \ll q_{i}^{* 0} .
$$

L'équation (47) montre que le poids relatif du mode d'ordre $\nu$ décroît comme $\nu^{4}$. La différence de comportement par rapport au cas précédent tient à ce que l'intensité pompante est insuffisante pour « orienter les modes de diffusion supérieurs $»$.

Dans tous les cas $\gamma_{i}^{(\nu)} / \gamma_{i}^{(1)}$ décroît très vite quand $\nu$ croît. Par suite, les deux premiers modes sont en pratique seuls observables. Il en résulte aussi que la condition (44) définissant l'intensité lumineuse pompante nécessaire pour réaliser une orientation uniforme sur le volume $V$, ne porte en pratique que sur les deux premiers modes et peut s'écrire $1 / k_{i} T_{p} \gg 1 / \tau_{i}^{(1)}, 1 / \tau_{i}^{(2)}$.

2. GAS D'Une « ORIENTAtion NON GONFINÉE 》 $\mu_{i} R \ll 1$. - Remarquons tout d'abord que la zone de pression envisagée ici est d'autant plus grande que l'enduit est de meilleure qualité.

Pour le premier mode, nous avons trouvé que dans toute la zone de pression telle que $\mu_{i} R \ll 1, T_{i}^{(1)}$ ne dépend pas de la pression et est égal à la valeur de la constante de temps en l'absence de gaz $T_{i}$. Sur la courbe qui représente $1 / T_{i}^{(1)}$ en fonction de $p$ dans le cas d'un enduit, ceci se traduit par le fait que la tangente à l'origine est horizontale (cf. fig. 3 , courbe $b$ ).

D'autre part, on trouve que le poids des modes $\nu>1$ est complètement négligeable. Ce résultat a été démontré (§ III.4) en supposant l'intensité lumineuse pompante uniforme sur le volume $V$, mais on peut prouver qu'il est encore vrai si l'intensité lumineuse pompante varie d'un point à un autre de la cellule.

3. CAS INTERmÉDIAIRE $\mu_{i} R \approx 1$. - Dans ce domaine de pression, il est nécessaire d'évaluer $T_{i}^{(1)}$ à partir de l'équation exacte (42) en utilisant par exemple le tableau I où nous avons également indiqué en fonction de $\mu_{i} R$ le pouvoir tampon du gaz :

$$
T_{i}^{(1)} / T_{i}=3 \mu_{i} R /\left(h_{1}\right)^{2} .
$$

La figure 4 donne en coordonnées réduites la courbe de variations de $1 /\left(T_{i}^{(1)}\right)^{*}$ en fonction de $p^{*}$ :

$$
\begin{gathered}
1 /\left(T_{i}^{(1)}\right)^{*}=T_{i} / T_{i}^{(1)} \\
p^{*}=\frac{3 \alpha_{i}}{2\left(2-\alpha_{i}\right)} \cdot \frac{R}{\lambda_{t}\left(p_{0}\right)} \cdot \frac{p}{p_{0}} .
\end{gathered}
$$




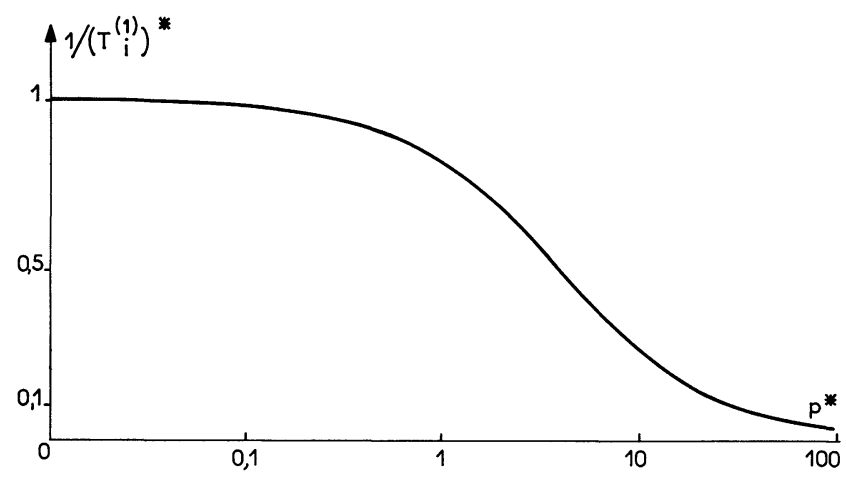

FIG. 4. - Variation de $1 / T_{i}^{(1)}$ en fonction de $\log p$ en coordonnées réduites (définies par les équations (48) et (49)).

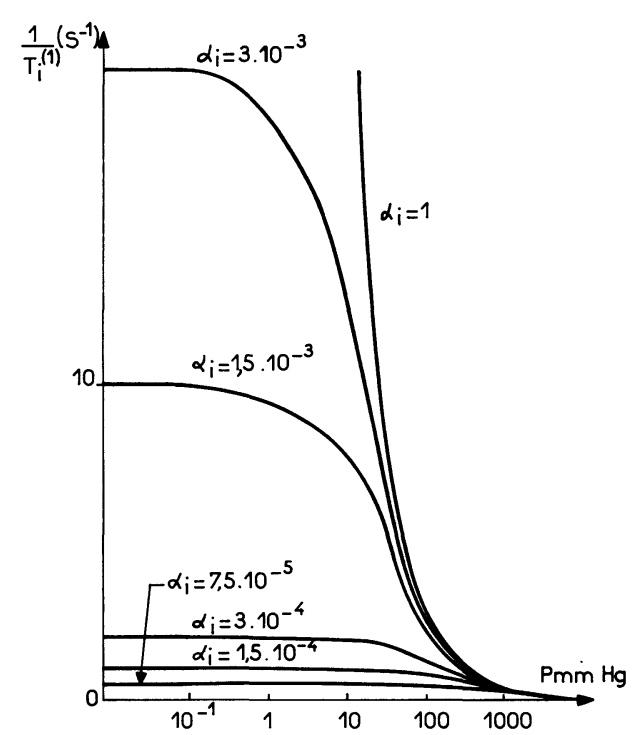

FIG. 5. - Variations de $1 / T_{i}^{(\mathbf{1})}$ en fonction de $\log p$ pour différents types d'enduits. Ces valeurs sont calculées pour une cellule de $3 \mathrm{~cm}$ de rayon, un coefficient de diffusion $D_{0}=0,3 \mathrm{~cm}^{2} / \mathrm{s}$ pour $p_{0}=1$ atm et des valeurs de $\alpha_{i}$ variant de $3 \times 10^{-3}$ à $7,5 \times 10^{-5}$.

Elle permet d'obtenir directement $1 / T_{i}^{(1)}$ en fonction de $p$ lorsqu'on se fixe un alcalin, un gaz rare, une qualité d'enduit, un rayon de cellule. La figure 5 représente en coordonnées usuelles plusieurs des courbes $1 / T_{i}^{(1)}=f(p)$ correspondant à différentes qualités d'enduit : on constate en particulier que la zone d'orientation « non confinée » se réduit de plus en plus quand $\alpha_{i}$ augmente.

B. Résultats concernant la relaxation sous l'effet simultané des processus $a$ et $b$. - Nous distinguons deux cas qui correspondent à ceux étudiés expérimentalement :

- cas des cellules à parois de verre nues ou très fortement contaminées, par des miroirs de métal alcalin $\left(\alpha_{i}=1\right)$;

- cas des cellules non contaminées avec enduit $\left(\alpha_{i} \ll 1\right)$.
1. Cellules a parois de verre nues. - Nous sommes alors, quel que soit $p$, dans le cas d'une orientation «" confinée » où les résultats concernant $T_{i}^{(v)}$ et $\gamma_{i}^{(v)}$ sont indépendants de l'observable envisagée (indépendants de $i$ ), car ils sont déterminés par le temps de diffusion des différents modes vers la paroi. Sous l'effet simultané des deux processus, les constantes de temps de relaxation $\tau_{i}^{(\nu)}$ sont donc données en fonction de $T^{(v)}$ (valeur commune des $T_{i}^{(v)}$ pour $\alpha_{i}=1$ ) par l'expression :

$$
\frac{1}{\tau_{i}^{(v)}}=\frac{1}{T^{(\nu)}}+\frac{1}{T_{i}^{\prime}}=D_{0} \frac{p_{0}}{p} \frac{\nu^{2} \pi^{2}}{R^{2}}+\frac{1}{T_{i}^{\prime}} .
$$

Aux faibles pressions où la relaxation sur la paroi est dominante (on peut négliger $1 / T_{i}^{\prime}$ ), on a :

$$
\frac{\tau_{i}^{(\nu)}}{\tau_{i}^{(1)}} \approx \frac{T_{i}^{(\nu)}}{T_{i}^{(1)}}=\frac{1}{\nu^{2}} .
$$

Aux pressions fortes, au contraire, la relaxation est dominée par les collisions sur le gaz et le temps de relaxation $\left(T_{i}^{\nu}=T_{i}^{\prime}\right)$ est inversement proportionnel à la pression. C'est la région où l'orientation est détruite sur le gaz, avant d'avoir le temps de diffuser, si bien que la même constante de temps $T_{i}^{\prime}$ apparaît sur tous les modes.

Dans la zone des pressions faibles et fortes et pour l'observable $Q_{i}$, la courbe $\Gamma_{i}^{(v)}$ représentant les variations de $1 / \tau_{i}^{(\nu)}$ en fonction de la pression s'obtient en faisant la somme des ordonnées de l'hyperbole $C^{(v)}$ (indépendante de l'observable $Q_{i}$ ) d'équation

$$
\frac{1}{T^{(v)}}=\frac{D_{0} p_{0} \pi^{2}}{R^{2}} \frac{v^{2}}{p}
$$

et de la droite $C_{i}^{\prime}$ (indépendante de $\nu$, mais dépendant de $\left.Q_{i}\right)$ d'équation

$$
\frac{1}{T_{i}^{\prime}}=\frac{N_{0} \beta_{i} \sigma v_{\mathrm{rel}}}{p_{0}} p .
$$

La figure 6 représente par exemple $\Gamma_{i}^{(1)}$. Pour obtenir $\Gamma_{i}^{(2)}$ (relatif à la même observable $Q_{i}$ ), il faudrait ajouter $4 / T^{(1)}$ à $1 / T_{i}^{\prime}$.

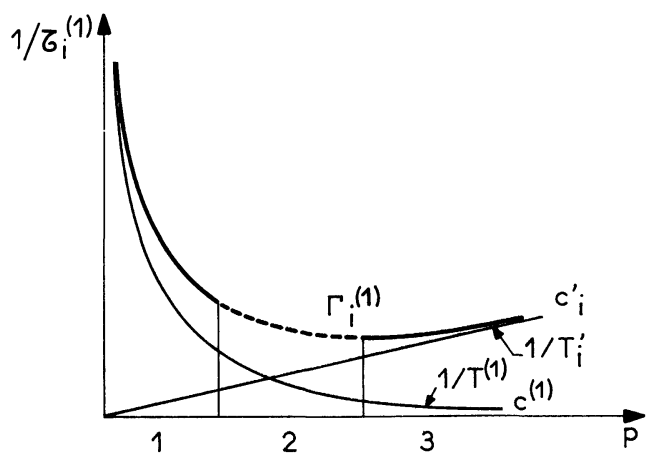

FIG. 6. - Variations de $1 / \tau_{i}^{(\nu)}$ en fonction de la pression dans le cas d'une cellule de verre nu. Les zones où la courbe $\Gamma$ est tracée en traits pleins sont celles où la relaxation de $\left\langle S_{z}\right\rangle$ se tait avec deux constantes de temps seulement. 
Les poids $\gamma_{i}^{(v)}$ sont ceux dont nous avons analysé les propriétés précédemment dans le cas d'une «orientation confinée »: ils dépendent de la répartition géométrique de $\left\langle Q_{i}\left(\mathbf{r}, t_{0}\right)\right\rangle$, donc des conditions géométriques de pompage. En pratique, $\gamma_{i}^{(v)}$ décroît très vite avec $\nu$ (d'autant plus que l'intensité pompante est plus faible) et on peut espérer détecter seulement les deux premiers modes.

Conclusions relatives à la relaxation de $\langle\mathbf{S} . \mathbf{I}\rangle$ et $\left\langle S_{z}\right\rangle$. - Pour la relaxation de $\langle\mathbf{S}$.I $\rangle$, nous nous attendons donc à observer, dans le cas général, deux constantes de temps $\tau_{H}^{(1)}$ et $\tau_{H}^{(2)}$ associées aux deux premiers modes de diffusion.

Pour $\left\langle S_{z}\right\rangle$ qui est une combinaison linéaire de deux observables $Q_{i}\left(Q_{e}\right.$ et $\left.I_{z}\right)$, on s'attend, dans le cas général, à une évolution avec 4 constantes de temps : $T_{n}^{(1)}$ et $T_{n}^{(2)}$ caractérisent la relaxation de $\left\langle I_{z}\right\rangle$ suivant les deux premiers modes de diffusion et $T_{e}^{(1)}$ et $T_{e}^{(2)}$ jouent le même rôle pour l'observable $\left\langle Q_{e}\right\rangle$ :

$$
\begin{aligned}
\left\langle S_{z}\right\rangle=\langle & \left.I_{z}\right\rangle_{0}\left(\gamma_{n}^{(1)} \mathrm{e}^{-t / \tau_{n}^{(1)}}+\gamma_{n}^{(2)} \mathrm{e}^{\left.-t / \tau_{n}^{(2)}\right)}\right. \\
& +\left\langle Q_{e}\right\rangle_{\mathbf{0}}\left(\gamma_{e}^{(1)} \mathrm{e}^{-t / \tau_{e}^{(1)}}+\gamma_{e}^{(2)} \mathrm{e}^{-t / \tau_{e}^{(2)}}\right) .
\end{aligned}
$$

Le rapport $\gamma_{n}^{(2)} / \gamma_{n}^{(1)}=\gamma_{e}^{(2)} / \gamma_{e}^{(1)}$ dépend de la répartition de $\left\langle Q_{e}\right\rangle_{0}$ et de $\left\langle I_{z}\right\rangle_{0}$ dans le volume de la cellule, et doit varier lorsqu'on modifie les conditions géométriques du pompage (par exemple les dimensions géométriques du volume de vapeur éclairé par le faisceau pompant).

Le rapport des poids relatifs de $\tau_{n}^{(1)}$ et $\tau_{e}^{(1)}$ dépend surtout de la valeur de $\left\langle Q_{e}\right\rangle_{0} \mid\left\langle I_{z}\right\rangle_{0}$ à l'instant initial ; il est susceptible de varier avec certaines caractéristiques de pompage : intensité, polarisation, forme spectrale de la lumière pompante. La théorie prévoit que si $\left\langle S_{z}\right\rangle_{0}=1 / 2, \gamma_{e}^{(1)} / \gamma_{n}^{(1)}=1,34$ pour $I=3 / 2$ et 2,40 pour $I=5 / 2$.

Il est important de remarquer que le second membre de l'équation (50), somme de 4 exponentielles, en fait intervenir deux seulement aux pressions faibles et fortes :

- aux pressions faibles, ce sont $\tau_{n}^{(1)} \approx \tau_{e}^{(1)} \approx T^{(1)}$ et $\tau_{n}^{(2)} \approx \tau_{e}^{(2)} \approx T^{(2)}=T^{(1)} / 4$ avec pour poids relatif $\gamma_{n}^{(2)} / \gamma_{n}^{(1)}=\gamma_{e}^{(2)} / \gamma_{e}^{(1)}$. De la mesure de $T^{(1)}$ on peut déduire la valeur du coefficient de diffusion $D_{0}$. On rencontre ce cas dans la zone (1) de la figure 6 ;

- aux pressions fortes, ce sont $\tau_{n}^{(1)} \approx \tau_{n}^{(2)} \approx T_{n}^{\prime}$ et $\tau_{e}^{(1)} \approx \tau_{e}^{(2)} \approx T_{e}^{\prime}$ avec pour poids relatif $\gamma_{n}^{(1)} / \gamma_{e}^{(1)}$. Des mesures de $T_{n}^{\prime}$ et $T_{e}^{\prime}$, on peut déduire les sections efficaces de désorientation relatives aux observables $\left\langle I_{z}\right\rangle$ et $\left\langle Q_{e}\right\rangle: \sigma_{n}$ et $\sigma_{e}$. De leur rapport, on peut déduire quelques conclusions relatives au spectre de Fourier de la perturbation produisant la relaxation (zone 3 de la figure 6).

2. Cellules avec enduit. - Ge cas correspond à $\alpha_{i} \ll 1$ et à des temps de relaxation très longs,
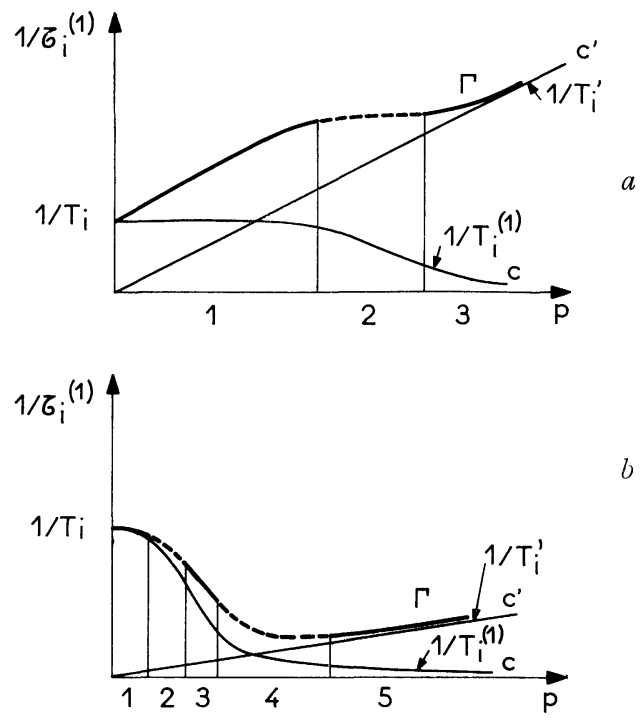

FIG. 7. - Variations de $1 / \tau_{i}^{(v)}$ en fonction de la pression dans le cas d'une cellule recouverte d'un enduit. Les zones où la courbe $\Gamma$ est tracée en traits pleins sont celles où la relaxation de $\left\langle S_{z}\right\rangle$ se fait avec deux constantes de temps seulement.

même en l'absence de gaz. La figure 7 représente la courbe $\Gamma$ donnant les variations de $1 / \tau_{i}^{(1)}$ en fonction de la pression du gaz tampon. Les courbes $\mathrm{C}$ et $\mathrm{C}^{\prime}$ représentent $1 / T_{i}^{(1)}$ et $1 / T_{i}^{\prime}$ en fonction de $p$. Ici encore, la courbe $\Gamma$ s'en déduit en faisant la somme des ordonnées des points de $\mathrm{C}$ et $\mathrm{C}^{\prime}$ ayant même abscisse. Il est clair que maintenant la courbe $C$ n'est plus une hyperbole, elle a la forme d'une courbe en cloche, d'ordonnée à l'origine $1 / T_{i}$ (donc différente pour les différentes observables $Q_{i}$ ). Nous pouvons distinguer deux cas :

- celui de la figure 7 a correspond à un enduit de très bonne qualité ou à un gaz très désorientant ;

- celui de la figure $7 b$ correspond à un enduit de qualité médiocre ou à un gaz très peu désorientant.

De manière générale, en se bornant aux deux premiers modes de diffusion, une observable $Q_{i}$ relaxe avec deux constantes de temps distinctes $\tau_{i}^{(1)}$ et $\tau_{i}^{(2)}$ (le poids de $\tau_{i}^{(v)}$ pour $\nu>2$ étant généralement négligeable dans les conditions d'observation). On doit donc s'attendre à observer deux constantes de temps sur $\langle\mathbf{S} . \mathbf{I}\rangle\left(\tau_{H}^{(1)}\right.$ et $\left.\tau_{H}^{(2)}\right)$ et quatre sur $\left\langle S_{z}\right\rangle\left(\tau_{n}^{(1)}\right.$, $\tau_{n}^{(2)}, \tau_{e}^{(1)}$ et $\left.\tau_{e}^{(2)}\right)$. Cependant, le nombre de constantes de temps observées sur $\left\langle S_{z}\right\rangle$ peut en pratique se réduire à deux :

- soit parce que le poids de $\tau_{i}^{(2)}$ est négligeable devant celui de $\tau_{i}^{(1)}$; c'est le cas d'une orientation « non confinée » $\left(\mu_{i} R \ll 1\right)$;

- soit parce que $\tau_{i}^{(1)}$ et $\tau_{i}^{(2)}$ sont indépendants de $i$; nous verrons que c'est le cas d'une orientation " confinée » $\left(\mu_{i} R \gg 1\right)$ et d'un gaz peu désorientant;

- soit parce que $\tau_{i}^{(1)} \approx \tau_{i}^{(2)}$, c'est-à-dire aux fortes pressions où la relaxation sur le gaz domine. 
Nous allons envisager plus en détail ces trois cas de manière à situer sur les figures $7 a$ et $7 b$ les zones de pression correspondantes.

a) Dans la zone de basse pression : orientation « non confinée " (zone 1 des figures $7 a$ et $b$ où la courbe $C_{i}$ est pratiquement horizontale), on trouve que le poids de tous les modes d'ordre supérieur à 1 est négligeable. Seul le premier mode subsiste et la constante de temps correspondante $T_{i}^{(1)}$ tend vers $T_{i}$, temps de relaxation sur la paroi en l'absence de gaz. Il n'y a donc qu'une constante de temps $\tau_{i}$ par observable $Q_{i}$ :

$$
\frac{1}{\tau_{i}}=\frac{1}{\tau_{i}^{(1)}}=\frac{1}{T_{i}}+\frac{1}{T_{i}^{\prime}}
$$

On observera $\operatorname{sur}\left\langle S_{z}\right\rangle$ les deux constantes de temps $\tau_{e}^{(1)}$ et $\tau_{n}^{(1)}$.

$\beta)$ Dans la zone de l'orientation " confinée " $\left(\mu_{i} R \gg 1\right)$ (zone 3 de la figure $7 a$ et zones 3,4 et 5 de la figure $7 b$, zones où la pression est supérieure à celle du point d'inflexion de la courbe $\mathrm{G}$ ), les valeurs des constantes de temps $T_{i}^{(v)}$ (égales au temps de diffusion du centre à la paroi, pour les différents modes $v$ ) deviennent indépendantes de la qualité de l'enduit, donc de $i$ :

$$
\frac{1}{\tau_{i}^{(v)}}=\frac{1}{T^{(v)}}+\frac{1}{T_{i}^{\prime}}
$$

Suivant que la section efficace de désorientation (sur le gaz) pour l'observable $Q_{i}$ est petite ou grande, c'est le premier ou le deuxième terme du second membre de cette expression qui l'emporte :

- Si $1 / T_{i}^{\prime} \ll 1 / T^{(1)}, 1 / T^{(2)}$, chaque observable $Q_{i}$ relaxe avec deux constantes de temps distinctes $T^{(1)}$ et $T^{(2)}$ indépendantes de $i$. Dans ce cas, $\left\langle S_{z}\right\rangle$ relaxe aussi avec deux constantes de temps seulement, $T^{(1)}$ et $T^{(2)}$. La condition posée n'est réalisée que sur la figure $7 b$ dans la zone 3 .

- Si $1 / T_{i}^{\prime} \gg 1 / T^{(1)}, 1 / T^{(2)}$, chaque observable $Q_{i}$ relaxe avec une seule constante de temps $T_{i}^{\prime}$ et $\left\langle S_{z}\right\rangle$ relaxe avec les deux constantes de temps $T_{e}^{\prime}$ et $T_{n}^{\prime}$. Sur les figures $7 a$ et $7 b$, la condition posée est réalisée pour les très fortes pressions : zone 3 de la figure $7 a$ et zone 5 de la figure $7 b$.

En conclusion, les parties des courbes $\Gamma$ en traits pleins correspondent aux domaines de pression où $\left\langle S_{z}\right\rangle$ relaxe avec deux constantes de temps seulement. Ce sont les seuls où nous pouvons espérer interpréter les résultats expérimentaux de manière non ambiguë.

Nous remarquons que, suivant les conditions, les deux constantes de temps prévues pour l'évolution de $\left\langle S_{z}\right\rangle$ ont un sens totalement différent :

- ou bien, elles traduisent la relaxation d'une même observable Q suivant deux modes de diffusion différents, leur poids dépend alors de la géométrie du faisceau pompant (zone 3 de la figure $7 b$ ) ;

- ou bien, elles traduisent la relaxation de deux observables différentes $Q_{e}$ et $I_{z}$ sur l'enduit (zone 1 des figures $7 a$ et $7 b$ ) ou sur le gaz (zone 3 de la figure $7 a$ et zone 5 de la figure $7 b$ ). Dans ce cas, leur poids relatif doit changer si l'on fait varier l'intensité pompante (sans changer sa répartition géométrique dans le volume de la cellule).

\section{CONCLUSION}

Le calcul présenté ci-dessus permet d'obtenir l'équation d'évolution d'une observable sous l'effet simultané de la relaxation par collisions sur la paroi après diffusion dans le gaz et de la relaxation par collisions sur le gaz. Le calcul est valable pour une paroi totalement désorientante (verre nu, ou recouvert d'un miroir d'alcalin) et pour une perturbation désorientatrice en phase gazeuse aléatoire faible de type quelconque. Nous avons précisé la forme de l'équation d'évolution de $\left\langle S_{z}\right\rangle$ dans le cas particulier où cette interaction est de type magnétique.

Le calcul est aussi valable pour une paroi recouverte d'un enduit paraffiné (collisions faibles de type magnétique) si l'on suppose de plus que la perturbation désorientatrice en phase gazeuse est également une perturbation aléatoire faible de type magnétique.

Dans nos hypothèses, une observable quelconque peut toujours s'écrire sous la forme d'une combinaison linéaire d'un nombre fini d'observables particulières $Q_{i}$ dont l'évolution est une exponentielle pure dans les deux cas extrêmes suivants :

- pression de gaz nulle : la relaxation est due uniquement aux collisions sur la paroi;

- pression de gaz très forte : la relaxation est seulement due aux collisions sur le gaz.

Il résulte du calcul qu'aux pressions intermédiaires une observable $Q_{i}$ relaxe avec une infinité dénombrable de constantes de temps, chacune associée à un certain mode de diffusion des atomes vers la paroi. Le poids de chaque constante de temps dépend de la répartition géométrique de la grandeur physique associée à l'observable mesurée à l'instant initial à partir duquel on étudie la relaxation.

Nous avons supposé que le système atomique était préparé dans cet état initial par pompage optique avec un faisceau lumineux dont toutes les caractéristiques (intensité, polarisation, profil spectral) étaient les mêmes en tous les points du volume occupé par les atomes. Dans ces conditions, on s'attend à n'observer qu'au plus deux constantes de temps par observable $Q_{i}$ : celles qui sont associées aux deux premiers modes de diffusion de $Q_{i}$. Par suite, $\left\langle S_{z}\right\rangle$, combinaison linéaire de deux observables $Q_{i}$, relaxe en général avec quatre constantes de temps. Cependant, dans plusieurs cas que nous avons analysés en détail, le nombre de constantes de temps distinctes se réduit à deux; c'est seulement dans ces conditions que nous envisageons comme intéressante la confrontation des résultats théoriques et expérimentaux.

Nous tenons à remercier MM. J. Brossel et J.-P. Faroux pour les fructueuses discussions que nous avons eues au cours de notre travail.

Manuscrit reçu le 22 décembre 1966. 


\section{BIBLIOGRAPHIE}

[1] Brossei (J.), Mosser (J. M.) et Winter (M.), J. Physique Rad., 1955, 16, 814.

[2] Bouchiat (M.-A.), J. Physique, 1963, 24, 379 et 611.

[3] Bouchiat (M.-A.) et Brosseq, (J.), Phys. Rev., 1966, $147,41$.

[4] Abragam (A.), "The Principles of Nuclear Magnetism "(Oxford University Press).

[5] Bernheim (R. A.), J. Chem. Physics, 1962, 36, 135.

[6] Herman (R. M.), Phys. Rev., 1964, 136, A 1576.

[7] Herman (R. M.), Phys. Rev., 1965, 137, A 1062.

[8] Franzen (W.), Phys. Rev., 1959, 115, 850.

LEGOWSKI (S.), J. Chem. Physics, 1964, 41, 1313. Preobrazhenskir (N. G.) et Senina (S. V.), Optics and Spectroscopy, 1964, 17, 441. Voir également référence [14].

[9] TORrey (H. C.), Phys. Rev., 1956, 104, 563.
[10] GLASSTONE (S.) et EDLUND (M.), "The elements of nuclear reactor theory ", chap. V (D. Van Nostrand Company, 1952).

[11] Cohen-Tannoudji (C.), Diplôme d'Études Supérieures, Paris, 1956.

[12] Brewer (R. G.), J. Chem. Physics, 1963, 38, 3015.

[13] Aymar (M.), Bouchiat (M.-A.), Brossel, (J.), Phys. Letters, 1967, 24 A.

[14] Minguzzi (P.), Strrumia (F.), Violino (P.), Nuovo Cimento, 1966, à paraître.

[15] BouchiaT (M.-A.), Thèse, Paris, 1964, Public. Scient. et Techn. du Ministève de l'Air, 1965, N.T. 146.

[16] Courani (R.) et HilbERT (D.), "Methods of mathematical physics ", vol. 1, chap. V et VI (Interscience Publishers, 1953).

[17] SeEuws (F.), Thèse de $3^{\mathrm{e}}$ cycle, Paris, 1966. 